\title{
Use of Multi-Parallel Real-Time Quantitative PCR to Determine Blastocystis Prevalence and Association with Other Gastrointestinal Parasite Infection in a Rural Honduran Location
}

\author{
Kevin S. Naceanceno, ${ }^{1} \dagger$ Gabriela Matamoros, ${ }^{2} \dagger$ José Antonio Gabrie,,${ }^{2,3}$ Maria Elena Bottazzi, ${ }^{4}$ Ana Sanchez, ${ }^{2,3} \ddagger$ \\ and Rojelio Mejia ${ }^{1,4 *} \ddagger$ \\ ${ }^{1}$ Laboratory of Clinical Parasitology and Diagnostics, National School of Tropical Medicine, Baylor College of Medicine, Houston, Texas; \\ ${ }^{2}$ Instituto de Investigaciones en Microbiología, Universidad Nacional Autónoma de Honduras, Tegucigalpa, Honduras; ${ }^{3}$ Department of Health \\ Sciences, Brock University, St. Catharines, Canada; ${ }^{4}$ Department of Pediatric Tropical Medicine, Texas Children's Hospital Center for Vaccine \\ Development, National School of Tropical Medicine, Baylor College of Medicine, Houston, Texas
}

Abstract. To determine whether the presence of Blastocystis is associated with other gastrointestinal parasite infections, stool samples from 95 Honduran rural children were analyzed using multi-parallel quantitative real-time polymerase chain reaction (PCR) and Kato-Katz. Combined results detected the following prevalence: Blastocystis, 71.6\%; Trichuris trichiura, 63.2\%; Giardia lamblia, 40.0\%; Ascaris lumbricoides, 15.8\%; and Necator americanus, 4.2\%. Age was found associated with the quantity of both Blastocystis DNA $\left(r_{s}=0.524, P<0.001\right)$ and $T$. trichiura DNA in the stool (fg/ $\left.\mu \mathrm{L}\right)$ by quantitative PCR $\left(r_{s}=0.272, P<\right.$ 0.001 ). In addition, there was an association with $T$. trichiura and Blastocystis infection (odds ratio $[\mathrm{OR}]=4.72 ; 95 \% \mathrm{Cl}=1.83$, 12.20; $P<0.001$ ). These findings demonstrate a high prevalence of Blastocystis and other intestinal parasites in a rural location in Honduras.

In this study, a previously developed multi-parallel real-time quantitative PCR (qPCR) assay was used in combination with the Kato-Katz $(\mathrm{KK})$ technique to determine the association of Blastocystis with the presence of Ascaris lumbricoides, Ancylostoma duodenale, Necator americanus, Strongyloides stercoralis, Trichuris trichiura, Cryptosporidium spp., Entamoeba histolytica, and Giardia lamblia. ${ }^{1}$

The study was part of a larger investigation on soil-transmitted helminths (STHs), and a subsample of fecal samples from 95 children (mean age $=6.1$ years; range $=8$ months-13 years; $47.4 \%$ females) were analyzed for the present study. Children were residents of impoverished rural villages in and around La Hicaca, Department of Yoro, in northern Honduras. ${ }^{2}$ Children in these communities are treated annually and sometimes biannually with $400 \mathrm{mg} /$ day $\times 3$ days with albendazole. Stool samples' aliquots were stored in $70 \%$ ethanol and transported to Houston laboratories where they were stored at $-20^{\circ} \mathrm{C}$ for molecular diagnosis of intestinal parasites.

At collection, the KK method was used to determine the presence of STHs in stool samples; STH eggs were identified and the number of eggs per gram calculated. Kato-Katz smears were examined between 30 and 60 minutes of preparation. Quality control procedures were applied, with $10 \%$ of positive and $100 \%$ of negative smears reexamined by a different researcher immediately after the first reading.

DNA was extracted from $50 \mathrm{mg}$ of stool from each sample using MP FastDNA ${ }^{\mathrm{TM}}$ for Soil Kit (MP Biochemicals, Solon, $\mathrm{OH}$ ) according to the manufacturer's instructions for all parasites, except $T$. trichiura, which required an additional heating step. ${ }^{1}$

DNA extraction from the stool and qPCR methods were implemented as described by Mejia et al. ${ }^{1}$ Species-specific primers and probes were previously designed and tested for eight gastrointestinal (Gl) parasites. ${ }^{1}$ Primers for Blastocystis were designed for a segment of the small subunit (SSU) rRNA gene

*Address correspondence to Rojelio Mejia, Baylor College of Medicine, National School of Tropical Medicine, One Baylor Plaza, BCM 113, Houston, TX 77030. E-mails: rmejia@bcm.edu or rojelio. mejia@bcm.edu

†These authors contributed equally to this work.

$\ddagger$ These authors contributed equally to this work. with the ability to amplify DNA from subtypes 1-10, as published by Poirier et al. ${ }^{3}$ The sequence information for Blastocystis primers and probes (target region SSU RNA, GenBank Accession No. J02459) used in multi-parallel qPCR were as follows: forward primer sequence $\left(5^{\prime} \rightarrow 3^{\prime}\right)$ AGTAGTCATACGCTCGTCTCAAA, reverse primer sequence $\left(5^{\prime} \rightarrow 3^{\prime}\right)$ TCTTCGTTACCCGTTACTGC, probe sequence (6-FAM, 5' $\rightarrow 3^{\prime}$ ) CGTGTAAATCTTACCATTAGAGGA. Standards were prepared for Blastocystis according to the protocol already described for other species. ${ }^{1}$

Samples were run on a ViiA ${ }^{\mathrm{TM}} 7$ Fast Real-Time PCR System (Applied Biosystems, Waltham, MA). For quantification, plasmids containing target sequences were run in duplicate according to dilution. An exogenous internal control was used to investigate the efficiency of DNA extraction, by spiking stool samples and measuring cycle threshold. ${ }^{4}$

Because KK is not suitable for detecting protozoa, qPCR and KK diagnostic methods were only compared for STH infections. The combined results from $\mathrm{KK}$ and $\mathrm{qPCR}$ were considered as the standard of diagnosis (i.e., any positive test result, regardless of the technique used, was considered a truepositive result) for $A$. lumbricoides, $T$. trichiura, and $N$. americanus.

Overall, 12 research participants were negative for parasites (12.6\%). Taking into account both diagnostic methods, Blastocystis was the most common parasite detected in 68 positive samples $(71.6 \%)$, followed by 60 positives for $T$. trichiura (63.2\%), 38 for G. lamblia (40.0\%), 15 for $A$. lumbricoides (15.8\%), and four for $N$. americanus (4.2\%). No positive samples were detected for $A$. duodenale, Cryptosporidium spp., S. stercoralis, or E. histolytica (Table 1).

Polyparasitism was frequent among research participants: $22(23.2 \%)$ were positive for one parasite species, 28 (29.5\%) for two, $26(27.4 \%)$ for three, six (6.3\%) for four parasites, and one $(1.1 \%)$ for five species. Counts are displayed for combined and individual methods in Table 1. The median and range of the concentration of DNA $(f g / \mu L)$ from qPCR-positive samples are also displayed.

Distribution of the intensity (burden) of infection by KK and qPCR was determined for STHs according to the thresholds set by WHO for $A$. lumbricoides (i.e., 1-5,000 light infection; $>$ 5,000-50,000 moderate infection; and > 50,000, heavy 
TABLE 1

Combined results from KK and qPCR for five gastrointestinal parasites detected $(n=95)$

\begin{tabular}{lcccc}
\hline \multicolumn{1}{c}{ Parasite } & $\begin{array}{c}\text { No. positive by } \\
\text { multi-parallel qPCR (\%) }\end{array}$ & $\begin{array}{c}\text { No. positive by the } \\
\text { KK method (\%) }\end{array}$ & $\begin{array}{c}\text { No. positive by both } \\
\text { methods (\%) }\end{array}$ & $\begin{array}{c}\text { DNA concentrations (fg/nL) positive in } \\
\text { stool from qPCR, median (range) }\end{array}$ \\
\hline $\begin{array}{l}\text { Blastocystis } \\
\text { Helminths }\end{array}$ & $68(71.6)$ & - & - & $1.135(0.010-200.4)$ \\
Ascaris lumbricoides & $10(10.5)$ & $15(15.8)$ & $15(15.8)$ & $10.7(0.143-1,797.8)$ \\
Necator americanus & $2(2.1)$ & $2(2.1)$ & $4(4.2)$ & $0.108(0.066-0.151)$ \\
$\quad$ Trichuris trichiura & $43(45.3)$ & $53(55.8)$ & $60(63.2)$ & $0.025(0.001-0.370)$ \\
Protozoa & $38(40.0)$ & - & - & $18.0(0.080-4,756.0)$ \\
$\quad$ Giardia lamblia & & &
\end{tabular}

infection), T. trichiura (i.e., $1-10^{3}$ light infection; $10^{3}-10^{4}$ : moderate infection; and $\geq 10^{4}$, heavy infection), and $N$. americanus (i.e., 1-2,000 light infection; > 2,000-4,000 moderate infection; and $>4,000$ heavy infection). ${ }^{5}$ In terms of infection intensity, a moderate agreement was found between $\mathrm{KK}$ and qPCR for A. lumbricoides ( $\mathrm{K}=0.516 ; 95 \% \mathrm{Cl}=0.364,0.702 ; P<0.001)$, whereas for $T$. trichiura, the agreement was fair $(\mathrm{K}=0.389$; $95 \% \mathrm{Cl}=0.242,0.508 ; P<0.001)$.

Spearman's rank correlation coefficient $\left(r_{s}\right)$ indicated a positive correlation between age and the amount of Blastocystis DNA in stool $(\mathrm{fg} / \mu \mathrm{L})$ by qPCR $\left(r_{s}=0.524, P<0.001\right.$; Figure 1$)$. A weak positive correlation was noted between age and the amount of $T$. trichiura DNA in stool $(\mathrm{fg} / \mu \mathrm{L})$ by qPCR $\left(r_{\mathrm{s}}=0.272\right.$, $P=0.008)$. No similar correlation was seen for $G$. lamblia $\left(r_{\mathrm{s}}=\right.$ $0.142, P=0.169)$, $A$. lumbricoides $\left(r_{\mathrm{s}}=-0.040, P=0.701\right)$, or $N$. americanus (not available). There was an association between Blastocystis infection and $T$. trichiura $(\mathrm{OR}=4.72 ; 95 \% \mathrm{Cl}=1.83$, 12.20; $P<0.001$ ), but not with the other Gl parasites (Table 2).

Blastocystis spp. is gaining attention globally as one of the most widespread organisms dwelling in the human Gl tract, although its pathogenicity is still in dispute. The amount of parasite DNA in stool samples was quantified using a previously developed multi-parallel qPCR method for $A$. lumbricoides, A. duodenale, N. americanus, S. stercoralis, T. trichiura, Cryptosporidium spp., E. histolytica, G. lamblia, and Blastocystis spp. These data were compared with KK data for three of the nine species (A. lumbricoides, $T$. trichiura, and $N$. americanus). A high prevalence of Blastocystis $(71.6 \%)$ was noted in this population in comparison with other Latin American studies undertaken in Brazil (21\% and 28\%), Argentina (25\%), and Colombia (12.6\%). ${ }^{6-9}$

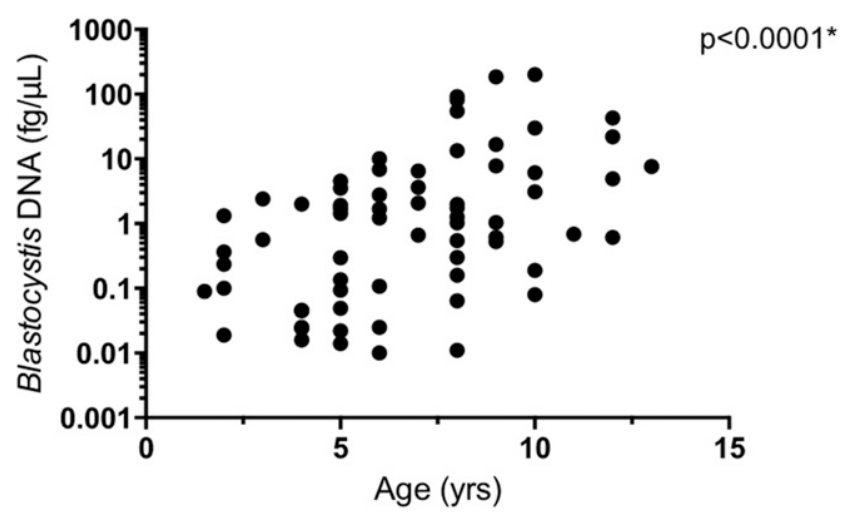

FIGURE 1. Spearman's rank correlation between Blastocystis burden and age. There was a correlation between age and the amount of Blastocystis DNA in stool (fg/ $\mu \mathrm{L})$ by quantitative PCR $\left(r_{s}=0.524\right.$, $P<0.001)$.
There was a correlation between age and Blastocystis infection (Figure 1). The correlation between age and prevalence of Blastocystis has been both present and absent in other similar studies. ${ }^{10-12}$ Some authors have suggested that Blastocystis is acquired via person-to-person transmission. ${ }^{13}$ In this case, increasing age would allow more opportunities for exposure. In addition, this organism is known to colonize individuals for several years, ${ }^{12}$ further increasing the likelihood that an older child would be infected if they were previously exposed. The association between $T$. trichiura infection and Blastocystis infection was noteworthy (Table 2). These two parasites have transmission sources in common, of which, contaminated soil and untreated water sources have been emphasized. ${ }^{14,15}$ Further studies characterizing water sources would be useful. Moreover, the fecal-oral route of infection likely has a major impact on our findings. In the study community, children likely are continuously reinfected. This is evident by the high STH prevalence despite regular deworming treatment. In addition to transmission routes, it is worth mentioning the role of the immune system in polyparasitism, particularly the interplay of STH and protozoa. ${ }^{16}$

Overall, this study's main contribution was to assess the prevalence and burden of Blastocystis, and its association with other Gl parasites in this rural pediatric population in Honduras, where specific studies on Blastocystis are lacking. Molecular methods of detection for this organism are in extensive use, and this study adds to the global body of data for an understudied region in Honduras. Because of the clinical and evolutionary relevance of the distribution of subtypes, the next step would be to map these subtypes in Honduras and other parts of Central America as previous authors have undertaken in Latin America. 9,17 This would delineate which subtypes are associated with helminth infections, if any, in this region. Understanding the interactions between common species of the GI tract such as Blastocystis and endemic Gl parasites is an essential step for improving human health.

TABLE 2

Statistical associations between other parasites and Blastocystis

\begin{tabular}{lcc}
\hline \multirow{2}{*}{$\begin{array}{c}\text { Gastrointestinal parasite } \\
\text { infection }\end{array}$} & OR $(95 \% \mathrm{Cl})$ & $P$-value \\
\cline { 2 - 3 } & & \\
\hline $\begin{array}{l}\text { Protozozoa } \\
\text { Giardia lamblia }\end{array}$ & $1.88(0.72-4.87)$ & 0.194 \\
Helminths & - & - \\
Necator americanus & $1.71(0.41,10.26)$ & 0.544 \\
Ascaris lumbricoides & $4.72(1.83,12.20)$ & $<0.001$ \\
\hline Trichuris trichiura &
\end{tabular}


In summary, we adapted a previously developed multiparallel qPCR assay to determine the prevalence and burden of various $\mathrm{Gl}$ parasites with fundamental demographic factors including gender, age, and infection of other known parasites of the Gl tract. Blastocystis was the most commonly detected organism $(71.6 \%)$. Also, the amount of Blastocystis DNA positively correlated with age in a single rural location in Honduras among a pediatric population.

Received November 27, 2019. Accepted for publication January 26, 2020.

Published online March 16, 2020.

Acknowledgment: We thank Patricia Bryan at Baylor College of Medicine for guidance regarding some statistical analysis.

Financial support: Research funding support for R. M. was provided by the U.S. Department of Health and Human Services, Health Resources and Services Administration for Baylor College of Medicine Center of Excellence in Health Equity, Training and Research (Grant No: D34HP31024). Funding was also provided by the Texas Children's Hospital Center for Vaccine Development and the National School of Tropical Medicine, Baylor College of Medicine.

Disclaimer: R. M. receives research funding from Romark Laboratories, LC, and this association did not impact this study.

Disclosure: The Honduras research team gathered informed consent. Internal Review Boards approved this research at the Universidad Nacional Autónoma de Honduras, Brock University, and Baylor College of Medicine. Every participant received a written result and a proper explanation about what the result meant. The Virginia Commonwealth University medical brigade visiting the communities during the study advised anthelminthic doses according to the infection, which was administered by a registered nurse of La Hicaca's health center. The applicable datasets have been made available in the supplemental files.

Authors' addresses: Kevin S. Naceanceno and Maria Elena Bottazzi, Department of Pediatrics, Baylor College of Medicine, Houston, TX, E-mails: naceance@outlook.com and bottazzi@bcm.edu. Gabriela Matamoros, Instituto de Investigaciones en Microbiología, Universidad Nacional Autónoma de Honduras, Tegucigalpa, Honduras, E-mail: gabrielamatamorosc@gmail.com. José Antonio Gabrie and Ana Sanchez, Department of Health Sciences, Brock University, St. Catherine's, Canada, E-mails: jgabrie@brocku.ca and asanchez@ brocku.ca. Rojelio Mejia, Baylor College of Medicine, National School of Tropical Medicine, Houston, TX, E-mail: rojelio.mejia@bcm.edu.

\section{REFERENCES}

1. Mejia R, Vicuna $Y$, Broncano N, Sandoval C, Vaca M, Chico M, Cooper PJ, Nutman TB, 2013. A novel, multi-parallel, real-time polymerase chain reaction approach for eight gastrointestinal parasites provides improved diagnostic capabilities to resource-limited at-risk populations. Am J Trop Med Hyg 88: 1041-1047.

2. Sanchez AL, Gabrie JA, Canales M, Rueda MM, Fontecha GA, Mason P, Bearman G, Stevens MP, 2016. Soil-transmitted helminths, poverty, and malnutrition in Honduran children living in remote rural communities. Hum Parasit Dis 8: 27-35.

3. Poirier P, Wawrzyniak I, Vivares CP, Delbac F, El Alaoui H, 2012. New insights into Blastocystis spp.: a potential link with irritable bowel syndrome. PLoS Pathog 8: e1002545.

4. Deer DM, Lampel KA, Gonzalez-Escalona N, 2010. A versatile internal control for use as DNA in real-time PCR and as RNA in real-time reverse transcription PCR assays. Lett Appl Microbiol 50: $366-372$.

5. WHO Expert Committee, 2002. Prevention and control of schistosomiasis and soil-transmitted helminthiasis. World Health Organ Tech Rep Ser 9: i-iv, 1-57, back cover.

6. Segui $R$ et al., 2018. Prevalence of intestinal parasites, with emphasis on the molecular epidemiology of Giardia duodenalis and Blastocystis sp., in the Paranagua Bay, Brazil: a community survey. Parasit Vectors 11: 490.

7. Malheiros AF, Stensvold CR, Clark CG, Braga GB, Shaw JJ, 2011. Short report: molecular characterization of Blastocystis obtained from members of the indigenous Tapirape ethnic group from the Brazilian Amazon region, Brazil. Am J Trop Med Hyg 85: 1050-1053.

8. Casero RD, Mongi F, Sanchez A, Ramirez JD, 2015. Blastocystis and urticaria: examination of subtypes and morphotypes in an unusual clinical manifestation. Acta Trop 148: 156-161.

9. Ramirez JD, Florez C, Olivera M, Bernal MC, Giraldo JC, 2017. Blastocystis subtyping and its association with intestinal parasites in children from different geographical regions of Colombia. PLoS One 12: e0172586.

10. Yaicharoen R, Sripochang S, Sermsart B, Pidetcha P, 2005. Prevalence of Blastocystis hominis infection in asymptomatic individuals from Bangkok, Thailand. Southeast Asian J Trop Med Public Health 36 (Suppl 4): 17-20.

11. Yaicharoen R, Ngrenngarmlert W, Wongjindanon N, Sripochang $\mathrm{S}$, Kiatfuengfoo R, 2006. Infection of Blastocystis hominis in primary schoolchildren from Nakhon Pathom province, Thailand. Trop Biomed 23: 117-122.

12. Scanlan PD, Stensvold CR, Rajilic-Stojanovic M, Heilig HG, De Vos WM, O'Toole PW, Cotter PD, 2014. The microbial eukaryote Blastocystis is a prevalent and diverse member of the healthy human gut microbiota. FEMS Microbiol Ecol 90: 326-330.

13. Rojas-Velazquez L et al., 2018. Genetic diversity and distribution of Blastocystis subtype 3 in human populations, with special reference to a rural population in central Mexico. Biomed Res Int 2018: 3916263.

14. Taamasri $P$, Mungthin $M$, Rangsin R, Tongupprakarn B, Areekul W, Leelayoova S, 2000. Transmission of intestinal blastocystosis related to the quality of drinking water. Southeast Asian $J$ Trop Med Public Health 31: 112-117.

15. Echazu A et al., 2015. Effect of poor access to water and sanitation as risk factors for soil-transmitted helminth infection: selectiveness by the infective route. PLoS Negl Trop Dis 9: e0004111.

16. Weatherhead J, Cortes AA, Sandoval C, Vaca M, Chico M, Loor S, Cooper PJ, Mejia R, 2017. Comparison of cytokine responses in Ecuadorian children infected with Giardia, Ascaris, or both parasites. Am J Trop Med Hyg 96: 1394-1399.

17. Ramirez JD et al., 2016. Geographic distribution of human Blastocystis subtypes in South America. Infect Genet Evol 41: 32-35. 